\title{
Adenomi ipofisari in età geriatrica: analisi dei risultati clinici e chirurgici in un'ampia casistica
}

\author{
Salvatore Cannavò ${ }^{1}$
}

Pubblicato online: 24 luglio 2019

(c) Springer Nature Switzerland AG 2019

Commento a:

Pituitary adenomas in elderly patients: clinical and surgical outcome analysis in a large series. A. Spina, M. Losa, P. Mortini.

Endocrine (2019)

https://doi.org/10.1007/s12020-019-01959-0

Questo studio riporta l'esperienza di un prestigioso gruppo neurochirurgico italiano nel trattamento chirurgico del tumore ipofisario in pazienti ultrasessantacinquenni. Si tratta certamente di un argomento di grande attualità e rilevanza in considerazione dell'incremento della sopravvivenza della popolazione generale e dell' aumento dell'incidenza di questa malattia, grazie non solo alla maggiore disponibilità di tecniche di imaging a elevata sensibilità (TC, RMN, ma anche PET, ecc.) ma, soprattutto, alle migliorate competenze cliniche degli specialisti endocrinologi e, più in generale, di molti operatori della sanità. D'altra parte, con l'età aumentano sia l'incidenza dei tumori ipofisari, soprattutto dei macroadenomi, che i rischi anestesiologici e, quindi, le possibili controindicazioni all'intervento chirurgico.

Nel loro studio, Spina e collaboratori hanno rivalutato retrospettivamente 336 pazienti (59\% maschi) con età $>65$ anni, nei quali erano stati diagnosticati fra il 1990 e il 2015 adenomi ipofisari non funzionanti (230 casi), acromegalia (57 casi), malattia di Cushing (39 casi), prolattinomi (5 casi) o adenomi TSH-secernenti (5 casi). Quasi 1'89\% dei tumori erano macroadenomi, mentre l'invasione del seno cavernoso o l'estensione nel seno sfenoidale era presente nel 31 e nel $15 \%$ dei casi, rispettivamente. In 21 pazienti si era verificata

S. Cannavò

cannavos@unime.it

1 Dipartimento di Patologia Umana dell'Adulto e dell'Età Evolutiva G. Barresi, Università di Messina, Messina, Italia l'apoplessia del tumore. Alterazioni del campo visivo erano state diagnosticate in 155 casi prima dell'intervento. Dal punto di vista anestesiologico, 69 pazienti erano stati classificati ASA I, 182 ASA II, 79 ASA III e solo 2 ASA IV. I pazienti sono stati suddivisi in 2 gruppi: il gruppo 1 composto da pazienti $<70$ anni, il gruppo 2 dagli over 70 . Dopo la chirurgia, il recupero anche parziale della funzione visiva è stato documentato nell' $85 \%$ dei pazienti, senza differenze fra i due gruppi, mentre un peggioramento si è verificato solo in 3 casi. Il $68 \%$ dei pazienti con adenomi secernenti ha raggiunto un adeguato controllo dell'ipersecrezione ormonale, senza significative differenze fra il gruppo 1 e il gruppo 2, ma con una percentuale di successo nettamente inferiore $(20 \%)$ nei prolattinomi. Il tasso di mortalità perioperatoria è risultato pari all' $1,5 \%$, ma nell' $80 \%$ dei casi il decesso si è verificato nel gruppo 2 . Complicanze chirurgiche maggiori o minori si sono verificate nell' $8 \%$ e in meno del $5 \%$ dei casi, rispettivamente, ugualmente ripartite fra i due gruppi. Il diabete insipido postchirurgico è insorto solo in 9 casi, mentre l'iponatremia transitoria si è verificata in 18 casi, di cui la metà hanno presentato valori circolanti di sodio $<125 \mathrm{mEq} / \mathrm{L}$ (iponatremia grave). Dopo la chirurgia, un nuovo deficit gonadotropinico è stato documentato nel $12 \%$ dei casi, di TSH nel 3\% e di ACTH nel 7\%. La recidiva dopo sola chirurgia o chirurgia più radioterapia si è verificata in 31 casi, mediamente dopo 46 mesi.

Gli autori concludono che, diversamente da quanto ritenuto comunemente, la chirurgia trans-sfenoidale rappresenta una terapia sufficientemente sicura ed efficace per i tumori ipofisari anche in pazienti anziani, da praticare ovviamente quando essa è realmente necessaria. 